\title{
Tipificación de las láminas de agua generadas por actividades extractivas del "Parque Regional en torno a los ejes de los cursos bajos de los ríos Manzanares y Jarama" (Madrid, España)
}

\author{
Neftalí Roblas \& Javier García-Avilés \\ Centro de Investigaciones Ambientales de la Comunidad de Madrid "Fernando González Bernáldez". C/ San \\ Sebastián 71, 28791 SOTO DEL REAL (Madrid), España.
}

\begin{abstract}
RESUMEN
El "Parque Regional en Torno a los Ejes de los Cursos Bajos de los Ríos Manzanares y Jarama", localizado en el sudeste de Madrid, tiene 123 ecosistemas acuáticos leníticos. Las actividades mineras de extracción de áridos o yeso originaron 110 y 1 de estas lagunas, respectivamente. La tipificación de las diferentes clases en que estos ecosistemas artificiales se pueden dividir es el principal objetivo del presente estudio. Esta clasificación podría ser el primer paso para alcanzar la gestión integral de estos cuerpos de agua en el territorio. Se seleccionaron nueve variables que resumen las principales caracteristicas ambientales de estos sistemas y sus alrededores. Fueron empleadas técnicas estadísticas multivariantes, obteniéndose una tipología de ocho clases de lagunas generadas por la actividad minera. La localización de estos ecosistemas en diferentes unidades fisiográficas, sus distancias desde el río, el grado de conservación de la vegetación envolvente, el área de la superficie, y la pendiente de las orillas fueron variables que, básicamente, definen las clases obtenidas. Así, podemos concluir que la diversidad de clases obtenida se debe a la evolución de las actividades mineras en el área de estudio y al diferente grado de recuperación alcanzado por cada uno de estos ecosistemas acuáticos artificiales.
\end{abstract}

Palabras clave: Tipificación, gravera, extracción de áridos, Jarama, Manzanares, Madrid.

\begin{abstract}
The "Parque Regional en Torno a los Ejes de los Cursos Bajos de los Rios Manzanares y Jarama", is a Nature Reserve located to the South-East of Madrid (Spain). There are 123 lagoons within its boundaries, origined by mining extraction activities for sand and gravel (110 lagoons) or gypsum (1 lagoon). The main aim of this study was to classify these artificial ecosystems as a first step to achieving the integrated management of lagoons within the whole park. Nine variables summarising the main environmental features of these systems and their surroundings were selected. Multivariate statistical techniques were performed, generating a typology of 8 lagoon classes. The location of these lagoons in different physiographic units, distance from the river, degree of conservation of the surrounding vegetation, surface area, and the slope of shores were the variables defining lagoon type. We can conclude that the diversity of lagoon types due changes in the mining activities in the study area and to the different degree of recovery of lagoons.
\end{abstract}

Keywords: Typification, gravel-pit lake, sand and gravel extraction, Jarama, Manzanares, Madrid.

\section{INTRODUCCIÓN}

El "Parque Regional en torno a los ejes de los cursos bajos de los ríos Manzanares y Jarama", creado en Junio de 1994, presenta una gran variedad de ecosistemas con destacados valores ambientales, entre los que figuran especialmente los humedales. Estos ecosistemas forman parte de un área cuya calidad ambiental está 


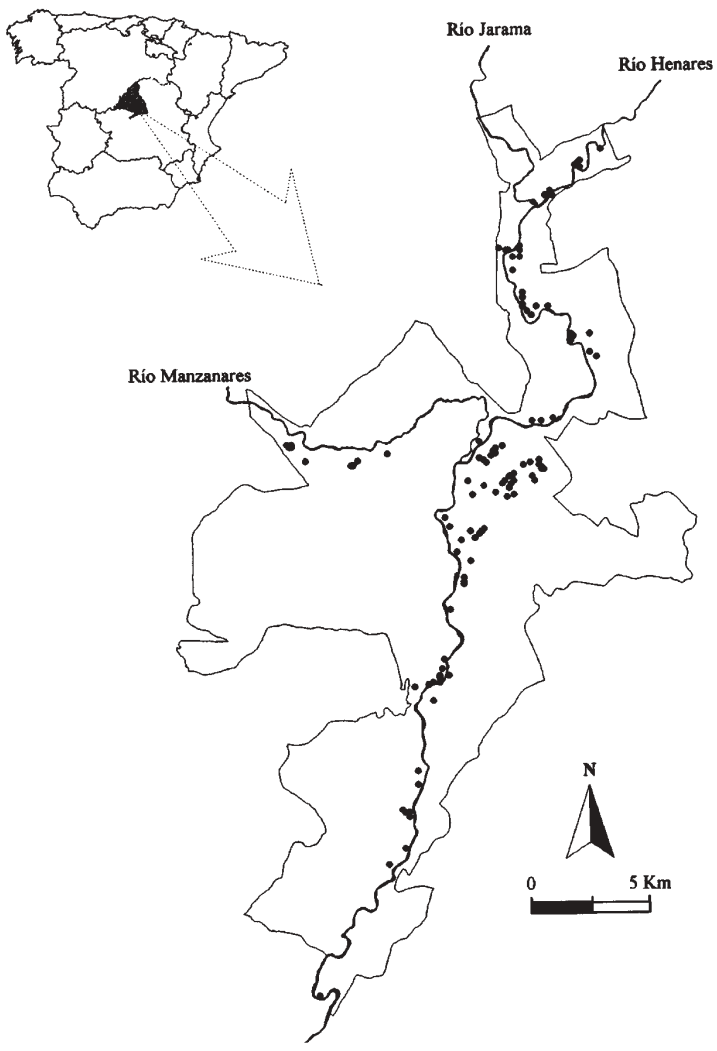

Figura 1. Mapa de localización del "Parque Regional en torno a los ejes de los cursos bajos de los ríos Manzanares y Jarama" y de las láminas de agua generadas por actividades extractivas. Location map of artificial lagoons in the Nature Reserve "Parque Regional en torno a los ejes de los cursos bajos de los ríos Manzanares y Jarama". definida por el contraste que supone la coexistencia de zonas de alto valor ecológico y la degradación producida por la actividad industrial, la inadecuada explotación de los recursos y factores derivados de su carácter periurbano (Comunidad de Madrid, 1994).

Este territorio alberga un total de 123 ecosistemas acuáticos leníticos, de los cuales 111 corresponden a láminas de agua generadas por actividades extractivas (Roblas \& García-Avilés, 1997). Dentro de estos ecosistemas acuáticos artificiales se encuentran algunas de las zonas húmedas más importantes de la región, incluidas en el Catálogo de Embalses y Humedales de la Comunidad de Madrid (Comunidad de Madrid, 1991), en cumplimiento de lo establecido en la "Ley sobre Protección de Embalses y Humedales" (Comunidada de Madrid, 1990).

El objetivo que se persigue con el presente trabajo consiste en diferenciar los distintos tipos de humedales artificiales que se han originado por actividades extractivas. Al establecer las diferentes clases de estos medios, cabe esperar que todos aquellos ecosistemas adscritos a cada uno de los tipos establecidos, tenga una respuesta similar su funcionamiento ecológico frente a los posibles usos o alteraciones. Con ello se facilita en gran medida su gestión y estudio, ya que se pueden centrar los esfuerzos de control e investi-

Tabla 1. Variables utilizadas y categorías asignadas para la tipificación de las láminas de agua generadas por actividades extractivas. Variables used and lagoon types derived from mining activities and recovery.

\begin{tabular}{lccc}
\hline VARIABLES & & CATEGORÍAS & \\
\hline & $\mathbf{1}$ & $\mathbf{2}$ & $\mathbf{3}$ \\
Litología & Arenas y Gravas & Zona de contacto & $\begin{array}{c}\text { Yesos y Margas Yesíferas } \\
\text { (Terciario Evaporítico) }\end{array}$ \\
Distancia Río & (Cuaternario Aluvial) & $250 \leq \mathrm{X}<1000 \mathrm{~m}$ & $\mathrm{X} \geq 1000 \mathrm{~m}$ \\
Superficie & $<250 \mathrm{~m}$ & $0,5 \leq \mathrm{X} \leq 2,5 \mathrm{ha}$ & $\mathrm{X}>2,5 \mathrm{ha}$ \\
Índice Costa* & $<0,5 \mathrm{ha}$ & $1,5 \leq \mathrm{X}<2,5$ & $\mathrm{X} \geq 2,5$ \\
Tipo de Cubeta & $\mathrm{X}<1,5$ & Mibtas \\
Granulometría & Encajadas & $1 \mathrm{~mm} \leq \mathrm{X}<5 \mathrm{~cm}$ & $\mathrm{X}^{3} \geq 5 \mathrm{~cm}$ \\
Vegetación Helófita & $<1$ mm & Media & Abundante \\
Vegetación Entorno & Ausente/Muy escasa & Cultivos & Matorral \\
Aislamiento & Sin vegetación & No aislada & Complejo lagunar \\
\hline
\end{tabular}

* Índice de Costa $=$ Perímetro $/ 2(\pi \text { Superficie })^{1 / 2}($ HUTCHINSON,1975) 
gación en determinados ejemplos de cada uno de los hábitats tipo descritos, sin tener que estar constantemente revisando todos y cada uno de los medios acuáticos del Parque.

Es necesario indicar que la tipificación que aquí se plantea es una clasificación básica que, junto con las aportaciones de futuros estudios, podría servir para determinar una tipología más amplia y en detalle de los humedales del Parque.

El área de estudio queda restringida al territorio definido por la Ley 6/1994 como "Parque Regional en torno a los ejes de los cursos bajos de los ríos Manzanares y Jarama". (Comunidad de Madrid, 1994). Este espacio natural protegido, que ocupa una extensión aproximada de $300 \mathrm{~km}^{2}$, se encuentra ubicado en el Sureste de la Comunidad Autónoma de Madrid (Fig. 1), ocupando una banda de territorio en sentido NorteSur que recorre los cursos fluviales de tres de los ríos más importantes de la Comunidad de Madrid, fundamentalmente el río Jarama y en menor medida, el Manzanares y el Henares.

Es de destacar la fuerte influencia sobre este área que tiene la proximidad de la capital, Madrid, y la presencia de un gran número de municipios de marcado carácter industrial situados en el perímetro del espacio protegido. Esto ha generado tensiones entre la conservación y la explotación intensiva del territorio, siendo las vegas bajas del Manzanares y del Jarama un ejemplo paradigmático de sustitución acelerada de usos del suelo (García et al., 1996).

\section{METODOLOGÍA}

En primer lugar, la determinación y elección de las variables claves que condicionan el funcionamiento y organización de estos ecosistemas artificiales resulta de vital importancia. Los medios acuáticos estudiados forman un conjunto de ecosistemas con una génesis común (actividades mineras), y pertenecen mayoritariamente a una unidad ambiental con características homogéneas: la Vega Baja Fluvial. Debido a esta homogeneidad ambiental no resulta recomendable la tipificación de estos medios en base a criterios genéticos e hidrológicos, comúnmente utilizados para clasificar ecosistemas acuáticos (González Besteiro, 1992; Montes, 1993; García-Avilés, 1994; Alonso et al., 1995), que solo posibilitarían la diferenciación entre grandes grupos, siendo más apropiados para regiones más extensas o con mayores diferencias ambientales.

Por ello, se han considerado variables que sinteticen tanto las diferencias entre los humedales derivadas de las características propias de la explotación minera (recurso explotado, intensidad, etc.), como de los procesos de naturalización que se están produciendo ya sea directamente en el medio acuático o en su entorno. En total han sido seleccionadas 9 variables, cada una de las cuales se ha dividido en 3 clases o modalidades para los posteriores análisis estadísticos (Tabla 1).

Por un lado, se ha realizado un análisis de clasificación que define las diferentes categorías en que se pueden agrupar las lagunas estudiadas. Para ello, en base a una matriz de asociación calculada con el coeficiente de distancia Chi-cuadrado para variables cualitativas multiestado, se ha efectuado un análisis de clasificación jerárquico de tipo politético aglomerativo UPGMA (Sokal \& Michener, 1958) por medio del programa informático PATN (Belbin, 1992)

Por otra parte, para comprobar qué variables son las más significativas y definen mejor los

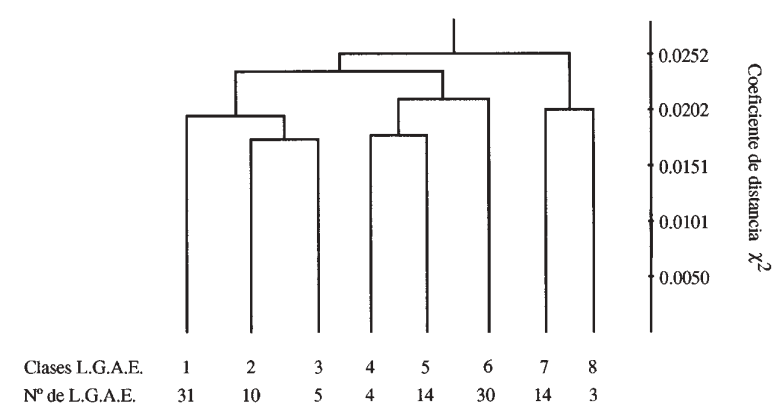

Figura 2. Dendrograma resultante del análisis de clasificación de las láminas de agua generadas por actividades extractivas (L.G.A.E.) del Parque, en el que se representan los grupos obtenidos y el número de lagunas que incluye cada uno de ellos. Dendrogram of the classification analysis of lagoons generated by mining activities in the Park, showing groups and the number of lagoons in each group. 
Tabla 2. Tipificación de las láminas de agua generadas por actividades extractivas del "Parque Regional en torno a los ejes de los cursos bajos de los ríos Manzanares y Jarama". Types of lagoon derived from extraction activities in the Nature Reserve "Parque Regional en torno a los Ejes de los Cursos Bajos de los Rios Manzanares y Jarama”.

\begin{tabular}{|c|c|c|c|}
\hline \multicolumn{2}{|c|}{ Tipología } & \multicolumn{2}{|l|}{ Nombre } \\
\hline Clase 1 & $\begin{array}{l}\text { Graveras del Río Henares } 1 \\
\text { Graveras del Río Henares } 2 \\
\text { Graveras del Río Henares } 3 \\
\text { Graveras del Río Henares } 4 \\
\text { Graveras del Río Henares } 5 \\
\text { Graveras del Río Henares } 6 \\
\text { Lagunas de la Presa del Río Henares } 1 \\
\text { Lagunas de la Presa del Río Henares } 2 \\
\text { Lagunas de la Presa del Río Henares } 3 \\
\text { Lagunas de la Presa del Río Henares } 4 \\
\text { Lagunas de la Presa del Río Henares } 5\end{array}$ & $\begin{array}{l}\text { Gravera El Negralejo } \\
\text { Graveras de Rivas } 2 \\
\text { Graveras de Rivas } 3 \\
\text { Graveras Corviam } 1 \\
\text { Graveras Corviam } 2 \\
\text { Graveras Corviam } 3 \\
\text { Lagunas del Campillo } 1 \\
\text { Lagunas del Campillo } 2 \\
\text { Lagunas del Campillo } 3 \\
\text { Graveras Soto Pajares } 5\end{array}$ & $\begin{array}{l}\text { Gravera Isla del Herrero } \\
\text { Graveras de San Martín de la Vega } 1 \\
\text { Graveras de San Martín de la Vega } 2 \\
\text { Graveras de San Martín de la Vega } 3 \\
\text { Graveras de San Martín de la Vega } 4 \\
\text { Graveras de San Martín de la Vega } 5 \\
\text { Graveras de San Martín de la Vega } 6 \\
\text { Graveras de San Martín de la Vega } 7 \\
\text { Gravera Ticosa } 1 \\
\text { Gravera Ticosa } 2\end{array}$ \\
\hline Clase 2 & $\begin{array}{l}\text { Graveras de Rivas } 4 \\
\text { Graveras de Rivas } 5 \\
\text { Lagunas de Las Madres } 4 \\
\text { Graveras de Arganda } 1\end{array}$ & $\begin{array}{l}\text { Graveras de Arganda } 2 \\
\text { Gravera Los Angeles } 3 \\
\text { Gravera Los Angeles } 1\end{array}$ & $\begin{array}{l}\text { Gravera Casas de San Medino } \\
\text { Gravera Casa de Castro } 3 \\
\text { Laguna de los Frailes }\end{array}$ \\
\hline Clase 3 & $\begin{array}{l}\text { Lagunas de Velilla } 3 \\
\text { Gravera Soto las Juntas }\end{array}$ & $\begin{array}{l}\text { Gravera Los Angeles } 2 \\
\text { Gravera Soto Gutiérrez } 2\end{array}$ & Gravera Soto de las Cuevas \\
\hline Clase 4 & $\begin{array}{l}\text { Graveras de Rivas } 1 \\
\text { Graveras de San Antonio } 3\end{array}$ & Graveras de San Antonio 4 & Graveras de San Antonio 5 \\
\hline Clase 5 & $\begin{array}{l}\text { Gravera Las Mantecas } 1 \\
\text { Graveras Camping Lagos } 1 \\
\text { Graveras Camping Lagos } 2 \\
\text { Laguna Muñoz } \\
\text { Laguna Villafranca }\end{array}$ & $\begin{array}{l}\text { Lagunas de Evencio Niño } 1 \\
\text { Lagunas de Evencio Niño } 2 \\
\text { Laguna Ulibarri } \\
\text { Graveras La Esperilla } 1 \\
\text { Graveras La Esperilla } 2\end{array}$ & $\begin{array}{l}\text { Graveras La Conejera } 4 \\
\text { Graveras La Conejera } 1 \\
\text { Graveras La Conejera } 2 \\
\text { Gravera Parque Tierno Galván }\end{array}$ \\
\hline Clase 6 & $\begin{array}{l}\text { Graveras Pioneer } 1 \\
\text { Graveras Pioneer } 2 \\
\text { Graveras Pioneer } 3 \\
\text { Graveras Pioneer } 4 \\
\text { Graveras Pioneer } 5 \\
\text { Graveras Pioneer } 6 \\
\text { Graveras Pioneer } 7 \\
\text { Gravera el Picón } \\
\text { Lagunas de Velilla } 2 \\
\text { Lagunas El Porcal } 1\end{array}$ & $\begin{array}{l}\text { Lagunas El Porcal } 3 \\
\text { Lagunas El Porcal } 4 \\
\text { Lagunas El Porcal } 5 \\
\text { Lagunas El Porcal } 6 \\
\text { Lagunas El Porcal } 7 \\
\text { Lagunas El Porcal } 8 \\
\text { Lagunas El Porcal } 9 \\
\text { Lagunas El Porcal } 10 \\
\text { Lagunas de Las Madres } 1 \\
\text { Lagunas de Las Madres } 3\end{array}$ & $\begin{array}{l}\text { Gravera Las Mantecas } 2 \\
\text { Graveras Soto Pajares } 2 \\
\text { Graveras Soto Pajares } 3 \\
\text { Graveras Soto Pajares } 4 \\
\text { Graveras Soto Pajares } 6 \\
\text { Gravera Maresa 1 } \\
\text { Lagunas de Ciempozuelos } 2 \\
\text { Lagunas de Ciempozuelos } 1 \\
\text { Gravera Casa de Castro } 2 \\
\text { Gravera El Charcón } 5\end{array}$ \\
\hline Clase 7 & $\begin{array}{l}\text { Lagunas de Velilla } 1 \\
\text { Lagunas El Porcal } 2 \\
\text { Lagunas de Las Madres } 2 \\
\text { Graveras Soto Pajares } 1 \\
\text { Graveras Soto Pajares } 7\end{array}$ & $\begin{array}{l}\text { Gravera Maresa } 2 \\
\text { Gravera Soto de la Chopera } 1 \\
\text { Gravera Soto de la Chopera } 2 \\
\text { Gravera Soto de la Chopera } 4 \\
\text { Gravera Casa de Castro } 1\end{array}$ & $\begin{array}{l}\text { Gravera El Charcón } 1 \\
\text { Gravera El Charcón } 2 \\
\text { Gravera El Charcón } 3 \\
\text { Gravera El Charcón } 4\end{array}$ \\
\hline Clase 8 & Gravera Maresa 3 & Gravera Soto Gutiérrez 1 & Gravera Soto de la Chopera 3 \\
\hline
\end{tabular}


diferentes grupos establecidos en el análisis anterior, se ha realizado un análisis de correspondencias múltiples.

\section{RESULTADOS}

El análisis de clasificación realizado agrupa las láminas de agua en ocho clases (Fig. 2 y Tabla 2), las cuales presentan las siguientes características:

Clase 1: Graveras sobre facies detríticas del Cuaternario aluvial (gravas y arenas), próximas al cauce fluvial (mayoritariamente a menos de $250 \mathrm{~m}$ ), con predominio de tamaños pequeño y medio (menos de 2,5 ha), que presentan abundante vegetación helófita, situadas en un entorno dominado por cultivos o matorral. En total son 31 las graveras incluidas en esta clase (Tabla 3).

Clase 2: Comprende 10 láminas de agua generadas en depósitos aluviales Cuaternarios, generalmente alejadas del cauce (distancia superior a 250 $\mathrm{m}$ ), de pequeña o mediana superficie (menores a $2,5 \mathrm{ha}$ ), con orillas poco sinuosas, estando situadas en zonas cultivadas u ocupadas por matorral.

Clase 3: Agrupa a 5 lagunas originadas por la explotación de áridos ubicadas sobre arenas y gravas (facies Cuaternarias aluviales), muy cercanas al cauce (menos de $250 \mathrm{~m}$ ), de gran tamaño (mayores a 2,5 ha) y que se encuentran aisladas. Presentan líneas de costa de baja sinuosidad, taludes en sus orillas ya sea de forma total o parcial, y vegetación litoral helófita abundante o media, apareciendo en un entorno destinado a uso agrícola o dominado por matorral (Tabla 3 ).

Clase 4: Está formada por 4 lagunas que han sido generadas directamente sobre materiales terciarios evaporíticos (yesos y margas yesiferas) o con fuerte influencia de éstos, situadas a menos de $600 \mathrm{~m}$ del curso fluvial, de superficie media a grande (siempre superior a $0,5 \mathrm{ha}$ ). Se localizan de forma aislada en entornos degradados (desprovisto de cobertura vegetal), y carecen generalmente de vegetación helófita.
Clase 5: La forman 14 lagunas de graveras localizadas sobre depósitos aluviales cuaternarios, mayoritariamente alejadas del cauce (a más de $1000 \mathrm{~m}$.), con superficies que generalmente exceden las 0,5 ha, situadas en ambientes degradados, y que aparecen de forma aislada.

Clase 6: Comprende un conjunto de 30 lagunas (Tabla 3), caracterizadas por encontrarse en facies del Cuaternario aluvial (arenas y gravas) y ocupar superficies mayores de 0,5 ha. Poseen vegetación litoral abundante o media, se localizan en entornos degradados (zonas activas, sin vegetación, etc.) y siempre forman complejos lagunares.

Clase 7: La forman un grupo de 14 láminas de agua generadas sobre terrazas y depósitos aluviales (gravas y arenas), de pequeño tamaño (inferiores a 0,5 ha), con cubetas encajadas, mayoritariamente sin vegetación helófita, y en entornos degradados carentes de vegetación. Forman siempre grupos de lagunas de mayor o menor entidad.

Clase 8: Agrupa a tan solo 3 lagunas que se localizan sobre facies del Cuaternario aluvial, próximas al cauce fluvial (menos de $250 \mathrm{~m}$. de distancia), de pequeño tamaño (superficie inferior a 0,6 ha), en las que no se ha desarrollado vegetación litoral, y situadas en un entorno carente de cobertura vegetal (Tabla 3 ).

Como ya se ha indicado anteriormente, con el fin de comprobar qué variables son las más significativas y definen mejor las clases establecidas, se ha realizado un análisis de correspondencias múltiples. En conjunto, los tres primeros ejes del análisis absorben el 65,39\% de la varianza total.

Observando la representación gráfica del análisis para los dos primeros ejes (Fig. 3), se aprecia que el eje I, que absorbe el $29,49 \%$ de la varianza, viene definido básicamente por la vegetación del entorno y la distancia del humedal al río. Así, los medios más cercanos a los cauces fluviales y con vegetación de los alrededores compuesta por matorral (Clase 1) se sitúan en el extremo positivo del eje, mientras que los ecosistemas más alejados del río y sin 
Tabla 3. Clases y características más relevantes de las láminas de agua generadas por actividades extractivas en el Parque. Classes and outstanding features of lagoons origined by mining activities in the Park.

\begin{tabular}{|c|c|c|c|c|c|}
\hline & Litología & $\begin{array}{l}\text { Características } \\
\text { Distancia al río }\end{array}$ & $\begin{array}{c}\text { Morfometría } \\
\text { (superficie/encajamiento) }\end{array}$ & $\begin{array}{c}\text { Vegetación } \\
\text { (helófitos/entorno) }\end{array}$ & $\mathbf{N}^{0}$ hum. \\
\hline Clase 1 & $\begin{array}{c}\text { Arenas y gravas } \\
\text { (Cuaternario Aluvial) }\end{array}$ & $<250 \mathrm{~m}$ & $<2,5$ ha/Muy diverso & Abundante/Cultivos-Matorral & 31 \\
\hline Clase 2 & $\begin{array}{c}\text { Arenas y gravas } \\
\text { (Cuaternario Aluvial) }\end{array}$ & $>250 \mathrm{~m}$ & $<2,5$ ha/Muy diverso & Variable/Cultivos-Matorral & 10 \\
\hline Clase 3 & $\begin{array}{c}\text { Arenas y gravas } \\
\text { (Cuaternario Aluvial) }\end{array}$ & $<250 \mathrm{~m}$ & $>2,5$ ha/Encajadas o mixtas & $\begin{array}{l}\text { Media o abundante/ } \\
\text { Cultivos-Matorral }\end{array}$ & 5 \\
\hline Clase 4 & $\begin{array}{l}\text { Yesos(Terciario } \\
\text { Evaporítico) y } \\
\text { Zona de Contacto }\end{array}$ & $<1000 \mathrm{~m}$ & $>0,5$ ha $/$ Diverso & Ausente/Ausente & 4 \\
\hline Clase 5 & $\begin{array}{c}\text { Arenas y gravas } \\
\text { (Cuaternario Aluvial) }\end{array}$ & $>1000 \mathrm{~m}$ & $>0,5$ ha/Muy diverso & Grupo heterogéneo/Ausente & 14 \\
\hline Clase 6 & $\begin{array}{c}\text { Arenas y gravas } \\
\text { (Cuaternario Aluvial) }\end{array}$ & Muy diversa & $>0,5 \mathrm{ha} /$ Diverso & Media o abundante/Ausente & 30 \\
\hline Clase 7 & $\begin{array}{c}\text { Arenas y gravas } \\
\text { (Cuaternario Aluvial) }\end{array}$ & Muy diversa & $<0,5 \mathrm{ha} /$ Encajadas & Ausente/Ausente & 14 \\
\hline Clase 8 & $\begin{array}{c}\text { Arenas y gravas } \\
\text { (Cuaternario Aluvial) }\end{array}$ & $<250 \mathrm{~m}$ & $<0,5$ ha/Muy diverso & Ausente/Ausente & 3 \\
\hline
\end{tabular}

vegetación en su entorno (Clase 5) se presentan en el extremo negativo.

En cuanto al eje II, que absorbe el $20,49 \%$ de la varianza, los parámetros que lo definen son el tipo de cubeta y la superficie. Los medios de menor tamaño se sitúan en el extremo positivo (por ejemplo, la Clase 2), mientras que los de mayor superficie, integrados además en complejos lagunares, se localizan en el extremo negativo del eje (Clase 6).

\section{DISCUSIÓN}

Debido al carácter artificial de los ecosistemas estudiados, tanto su funcionamiento como su estructura están fuertemente condicionados por la intervención humana, no respondiendo en gran medida a variables naturales sino a la evolución de los usos y técnicas de extracción minera, así como al tiempo de abandono y a las tareas de restauración llevadas a cabo en las lagunas.

Dado el origen antrópico de los ecosistemas acuáticos estudiados, serán aquellas variables más directamente dependientes de la actividad extractiva las que tengan mayor peso a la hora de caracterizar y diferenciar los distintos grupos de lagunas obtenidos en la tipificación. Este hecho se ve claramente reflejado en el análisis de ordenación realizado, en el que variables como la vegetación del entorno, la distancia al río o la superficie de la lámina de agua, poseen el mayor poder discriminante (Fig. 3). En los tres casos se trata de variables que se derivan de 
Símbolos

$$
\begin{array}{cc}
\hline & \text { L.G.A.E. Clase } 1 \\
\hline & \text { L.G.A.E. Clase } 2 \\
\text { - } & \text { L.G.A.E. Clase } 3 \\
* & \text { L.G.A.E. Clase } 4 \\
\hline & \text { L.G.A.E. Clase } 5 \\
- & \text { L.G.A.E. Clase } 6 \\
- & \text { L.G.A.E. Clase } 7 \\
\hline & \text { L.G.A.E. Clase } 8
\end{array}
$$

II

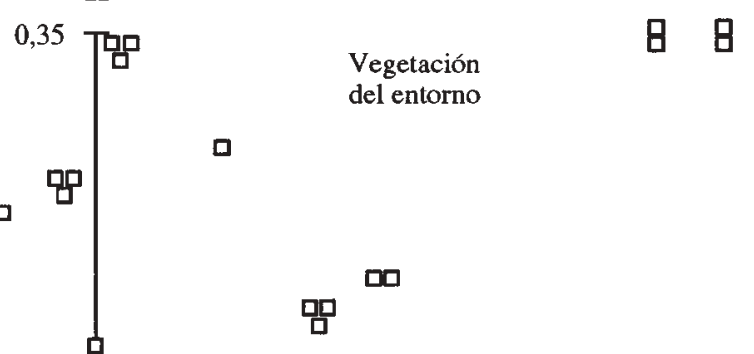

$\circ$

口

口 Aislamiento

$\Delta$

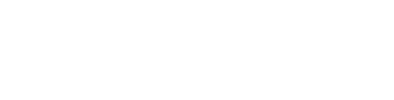

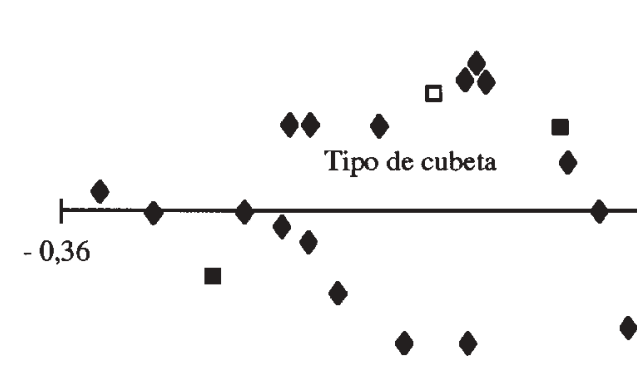

$\circ$

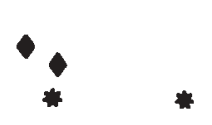

Superficie

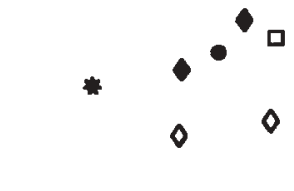

tat

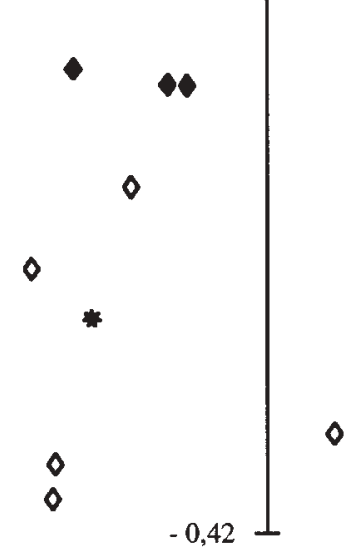

口

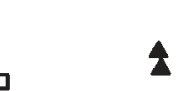

$\mathbf{t}$

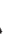

व $\quad \circ$
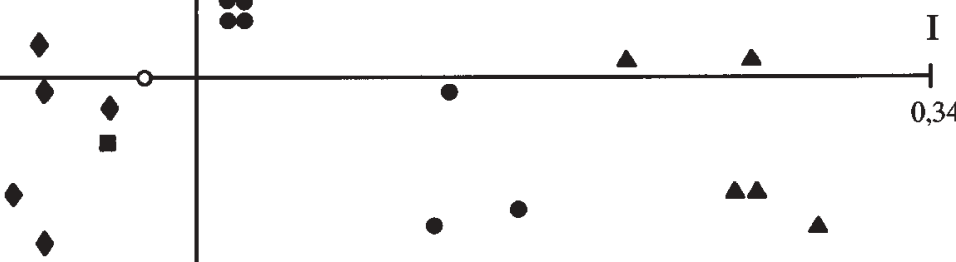

$\bullet$
$\Delta$

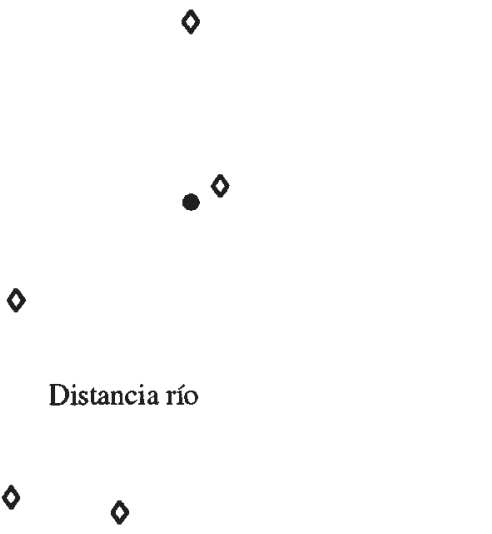

Figura 3. Distribución de las láminas de agua generadas por actividades extractivas (L.G.A.E.) y de las principales variables utilizadas en su tipificación, sobre el plano formado por los dos primeros ejes del análisis de correspondencias múltiples. Distribution of lagoons origined by mining activities and main variables used in their classification, on the plane of the two first axes of the multiple correspondence analysis. 
las características de las explotaciones mineras que han dado origen a los medios acuáticos estudiados, y que definen en gran medida las clases obtenidas (Tabla 3). En el caso de la vegetación del entorno, la ubicación de láminas de agua en zonas cultivadas o de matorral, refleja de manera clara el abandono de la actividad extractiva. Por otro lado, la distancia al río reflejará el tipo de materiales explotados y la unidad geomorfológica sobre la que se lleva a cabo la explotación; gravas y arenas de la Vega Fluvial, materiales yesíferos de Campiña, o gravas y arenas en las zonas de transición (Rampa). Por ello, las lagunas generadas por extracciones de áridos (arenas y gravas) de mayor magnitud se encontrarán próximas a los cauces fluviales. La superficie por su parte, constituye un fiel reflejo de la importancia de la explotación.

Como se observa en el dendrograma obtenido en el análisis de clasificación (Fig. 2), las clases 7 y 8 son las primeras en diferenciarse del resto de los grupos. Las lagunas que forman estas dos clases presentan como principales características, por un lado, la ausencia de vegetación tanto helófita como en su entorno, y por otra parte, superficies inferiores a 0,5 ha. Estas características diferencian a estos medios del resto de láminas de agua generadas sobre arenas y gravas del Cuaternario Aluvial (Tabla 3). La situación contraria en cuanto a la vegetación presente en los humedales y su entorno, definida por lagunas ubicadas en zonas destinadas a cultivos o con matorral en su entorno, y que presentan vegetación helófita media o abundante, está representada por las clases 1, 2 y 3 (Tabla 3). Parece claro que el primer grupo (clases 7 y 8 ), corresponde a lagunas en zonas activas de explotación, y por lo tanto degradadas, mientras que las segundas aparecen en zonas de actividad minera abandonada, por lo presentarán un mejor estado de conservación y mayor naturalización. Este hecho se confirma al comprobar que la mayoría de los complejos lagunares incluidos en "Zonas de Reserva Natural o Integral" dentro del Parque Regional por sus altos valores naturales, se encuentran incluidas en los clases 1,2 o 3, y especialmente en la primera de ellas. Este es el caso de las Graveras del río Henares, El Campillo o San Martín de la Vega (Comunidad de Madrid, 1994).

La distancia al río es otra de las variables con un fuerte peso a la hora de determinar los diversos tipos de lagunas presentes en la zona de estudio. Este factor posee una gran importancia en los ecosistemas estudiados, ya que de él van ha depender en gran medida los flujos subterráneos que se establecen entre las lagunas, el río y las aguas subválveas asociadas a éste, y el acuífero Cuaternario (Alonso et al., 1995). Una expresion de la relevancia de este complejo sistema hidrodinámico y su papel sobre las características del humedal viene dada por la relación entre la distancia al cauce y parám que definen el estado trófico de las lagunas. Así, según Montes (1993) existen fuertes relaciones entre la variable aquí considerada y el grado de eutrofización expresado como la concentración de clorofila $a$ y la concentración de fósforo disuelto.

De entre los 8 tipos de ecosistemas acuáticos generados por actividades extractivas que han sido definidos, la clase 5 se caracteriza por albergar a las lagunas más alejadas de los cauces fluviales (Tabla 3). Estas tienen su origen en antiguas explotaciones de gravas y arenas de pequeña entidad ubicadas en las Terrazas Altas del Cuaternario Aluvial. Por el contrario, las clases 1,3 y 8 , están formadas por lagunas muy próximas a los cauces (Tabla 3), habiendo sido generadas en la llanura de inundación del río o en terrazas bajas de la Vega Fluvial. En último término, es necesario señalar la especial situación de las lagunas pertenecientes a la clase 4. A priori sería lógico pensar que estas lagunas deberían aparecer alejadas de los cauces fluviales, ya que, o han sido generadas sobre formaciones de yesos, o en zonas de contacto entre estos materiales y facies del Cuaternario Aluvial. Sin embargo, la geomorfología de la zona de estudio, caracterizada por un fuerte encajamiento de los valles fluviales de los ríos Manzanares y Jarama que ha dado lugar a los denominados "cortados yesíferos", hace posible la génesis de estos humedales a escasa y media distancia de estos ríos. 
La superficie de las láminas de agua representa otra de las variables que mejor definen a los grupos de lagunas obtenidas en la tipificación. En la clase 6 se incluyen los grandes complejos lagunares del Parque originados por las explotaciones de áridos (gravas y arenas) más importantes. En éstos se encuentran láminas de agua que llegan a superar las 70 ha, como en el caso de las graveras del Porcal (Montes, 1993). A éstas hay que sumar las 5 lagunas que componen la clase 3 (Tabla 3). Por otra parte, las lagunas de menor tamaño aparecen en las clases 7 y 8 (Tabla 3), habiendo sido generadas por pequeñas extracciones de áridos en zonas de explotación activas actualmente.

Otra de las variables que claramente depende de las características de la explotación es el tipo de cubeta. Para la mayor parte de las clases obtenidas este factor resulta heterogéneo, integrando tanto a medios que presentan la lámina de agua encajada por taludes, como otras con orillas tendidas. Únicamente la clase 7 está formada por humedales encajados en todos los casos. La existencia de taludes en las orillas constituye un factor clave en la naturalización de este tipo de ecosistemas artificiales, ya que condiciona la presencia de vegetación helófita. $\mathrm{Al}$ no existir una franja litoral somera en el caso de cubetas encajadas, resulta imposible el desarrollo de este tipo de vegetación.

En conclusión, es necesario destacar que, debido al carácter artificial de estos ecosistemas, es la intervención humana la que controla su evolución, existiendo una gran diversidad de casuísticas. Por ello, resulta necesario estudiar y gestionar de forma integrada el conjunto de humedales incluidos en el Parque Regional, ya que medidas a adoptar apropiadas en algunos casos, pueden no serlo en otros en función de las características concretas de cada tipología.

\section{BIBLIOGRAFÍA}

ALONSO, C., I. GONZÁLEZ, A. ORDEJÓN \& L. E. PAREDES. 1995. Sectorización ambiental y caracterización de láminas de agua generadas por acti- vidades extractivas del Parque Regional en torno a los ejes de los cursos bajos de los ríos Manzanares y Jarama. Directrices de uso y recuperación. E.T.S.I. de Montes. V Master de Evaluación y Corrección de Impactos Ambientales.

BELBIN, L. 1992. PATn, Pattern Analysis Package. Division of Wildlife and Ecology, CSIRO. Australia.

COMUNIDAD DE MADRID. 1990. Ley 7/1990, de 28 de junio, de Protección de Embalses y Zonas Húmedas de la Comunidad de Madrid. B.O.C.M., 234: 28349-28352.

COMUNIDAD DE MADRID. 1991. Acuerdo de 10 de octubre de 1991, del Consejo de Gobierno, por el que se aprueba el Catálogo de Embalses y Humedales de la Comunidad de Madrid. B.O.C.M., 257: 4-6.

COMUNIDAD DE MADRID. 1994. Ley 6/1994, de 28 de junio, sobre el Parque Regional en torno a los ejes de los cursos bajos de los ríos Manzanares y Jarama. B.O.C.M., 163: 2-22.

GARCÍA-AVILÉS, J. 1994. Ecosistemas acuáticos leníticos del Parque Regional de la Cuenca Alta del Manzanares. Inventario y tipificación. Centro de Investigaciones Ambientales de la Comunidad de Madrid "Fernando González Bernáldez", Soto del Real. Serie Documentos $n^{\circ} 13.110$ pp.

GARCÍA, R., I. GONZÁLEZ, A. ORDEJÓN \& C. ALFONSO. 1996. Evolución del paisaje rural en la vega baja del Jarama (Madrid). Actas de las VIII Jornadas Sobre el Paisaje. La conservación del paisaje rural: 173-183.

GONZÁLEZ BESTEIRO, A. 1992. Limnología de las formaciones palustres situadas sobre el acuifero de Madrid. Análisis de las relaciones entre aguas superficiales y subterráneas. Tesis doctoral. Facultad de Ciencias, Universidad Autónoma de Madrid. 334 pp. HUTCHINSON, G. E. 1975. A Treatise on Limnology. Vol. I, Part. 1. Geography and Physics of Lakes. New York: John Wiley \& Sons.

MONTES, C. (dir.) 1993. Estudio de los valores ambientales existentes en las láminas de agua generadas por actividades extractivas en la Comunidad de Madrid. Agencia de Medio Ambiente, Comunidad de Madrid; Departamento Interuniversitario de Ecología, Univ. Autónoma de Madrid. 234 pp.

ROBLAS, N. \& J. GARCÍA-AVILÉS. 1997. Valoración ambiental y caracterización de los ecosistemas acuáticos leníticos del Parque Regional en torno a los ejes de los cursos bajos de los ríos 
Manzanares y Jarama. Centro de Investigaciones Ambientales de la Comunidad de Madrid "Fernando González Bernáldez", Soto del Real. Serie Documentos no 24. 128 pp.
SOKAL, R. R. \& C. D. MICHENER. 1958. A statistical method for evaluating systematic relationships. Univ. Kansas. Science Bulletin, 38: 1409-1438. 